\title{
Surgical infections at a regional hospital in Gauteng: reasons for delay to care and profile of pathology
}

\author{
N Patel ${ }^{1}$, P Naidoo², G Candy ${ }^{1}$, CJ Kruger ${ }^{3}$ \\ 1 Department of General Surgery, Faculty of Health Sciences, University of the Witwatersrand \\ 2 Department of Medicine, RK Khan Regional Hospital, Chatsworth, KwaZulu-Natal \\ 3 Department of Surgery, Edenvale General Hospital, Johannesburg, Gauteng
}

Corresponding author: N Patel (niravpatel44@gmail.com)

\begin{abstract}
Background: Present on arrival infection is a common indication for admission of surgical patients initially managed at primary care level. We aimed to describe the demographic and disease profile of patients presenting with infection requiring surgical management, describe determinants of patients' health-seeking behaviour, and identify barriers to care.

Methods: A prospective descriptive questionnaire-based study was conducted at Edenvale General Hospital between February 2014 and October 2016. Minors were excluded.

Results: Eighty-nine patients participated. Abscesses (26\%), diabetic foot (22\%), and cellulitis (16\%) were the commonest categories of infection necessitating admission. The majority of patients were South African (88\%), Black African (82\%), males (58\%), without medical aid (99\%), who were not formally employed (58\%), were from poor households $(74 \%)$, inhabited some form of formal housing $(90 \%)$, were in charge of decisions regarding personal health (80\%), and first sought help at the primary care level (71\%). Delay to presentation was noted in $69 \%$ of patients, and delay to referral in $46 \%$. Age, race, history of diabetes, and main source of monthly income were significant variables in delayed presentation $(p<0.05)$, and age and level of care on first contact in delayed referral $(\mathrm{p}<0.05)$ in the study sample. The most common reason for delay to presentation $(84 \%)$ and referral $(61 \%)$ was patients' belief that their problem would resolve spontaneously.

Conclusions: Patients' socio-economic status, past medical history, demographics, level of first contact with the health care system, and perceptions of their own health contributed to delays in seeking and receiving care in the study sample. These delays may be addressed by interventions that target the availability, accessibility, acceptability and affordability of health care services.
\end{abstract}

\section{Introduction}

Sepsis is a common indication for admission in surgical patients. Delay in the management of sepsis is strongly associated with increased rates of morbidity, mortality, and the overall cost of treatment. ${ }^{1,2,3}$ In low- and middle- income countries (LMICs) estimates suggest that access to essential surgery could avert $6 \%$ of all preventable deaths. ${ }^{4}$ In Africa, surgical conditions account for 25 million disability-adjusted life years (DALYs) annually. ${ }^{5}$ The majority of patients in Sub-Saharan Africa (SSA) with surgical problems fail to visit a health facility, are treated at poorly resourced facilities, or present with advanced disease. ${ }^{2,5,6,7,8}$ Infectious conditions constitute a significant portion of the surgical disease burden in LMICs, and the role of appropriate and timely surgery in the management of these diseases is increasingly being recognized. ${ }^{4}$

Impediments in seeking, reaching and receiving care may result in delays to care that often result in a significant economic and social burden on affected individuals, their families, society and health care systems. ${ }^{1,5,7-10}$ Barriers to care include educational level, health awareness, gender inequality, poverty, the geographical relationship between patients and health care facilities, confidence in and quality of available services, and the direct and indirect costs of accessing services. ${ }^{7-9,11}$

There is a paucity of data on barriers to care for patients with sepsis acquired in the out of hospital setting, requiring surgical management, in public hospitals in Gauteng. We aimed to describe the demographic and disease profile of patients with infection requiring surgical management, describe determinants of patients' health-seeking behaviour and identify barriers to care. This understanding is imperative to formulating appropriate interventions and strengthening the health care system.

\section{Methodology}

Ethical clearance was obtained from the University of the Witwatersrand Human Research Ethics Committee 
(M131182). A prospective descriptive questionnaire based study was performed at Edenvale General Hospital (EGH) between 1 February 2014 and 31 October 2016. Patients admitted to EGH surgical wards through EGH casualty, and those down referred from CMJAH, with infection present on admission requiring surgical management were included. Qualifying patients were identified by the surgical consultant at EGH. Minors were excluded. Convenience sampling was performed due to time, administrative and resource constraints. Questionnaires were completed by the surgical registrar (principal investigator) or medical officers placed within the ward. Questionnaires were administered in English and, where necessary, translators were utilised. For the purpose of this study, infection present on admission was defined as any source of sepsis acquired out of hospital setting requiring surgical intervention or management by a surgeon. The questionnaire focused on patient demographics, healthseeking behaviour, and utilisation of health care services. The questionnaire used questions developed for the Health, Environment and Development (HEAD) study undertaken by the Medical Research Council of South Africa. ${ }^{12}$ In particular, the questionnaire assessed barriers to care in terms of the: (i) availability, (ii) affordability, and (iii) acceptability and (iv) accessibility of health care services.

All patient data was anonymised and confidential. Participants provided written informed consent, participation was voluntary and no participant received material benefit. Descriptive statistics were generated using MS Excel ${ }^{\circledR}$. Statistical analysis was performed using the Student's t, ChiSquared and Fisher's Exact tests in $\mathrm{R}^{\circledR}$. Statistically significant differences between groups within categorical variables identified as significant by the Chi-Squared or Fischer's Exact tests were determined using logistic regression. These results are presented by means of an odds ratio (OR) and 95\% confidence interval (CI). Any patient presenting to a health care worker more than 48 hours after the onset of symptoms was considered a delayed presentation. Once assessed, any patient presenting for specialist care more than 24 hours after referral was considered a delayed referral. Level of significance was set at $\mathrm{p}<0.05$.

Edenvale General Hospital (EGH) is a regional hospital in the East of Johannesburg. EGH serves a population of approximately 3 million individuals from the suburbs of Edenvale, Lombardy, Alexandra, Tembisa, Linksfield and their surroundings. EGH is located within 15 kilometres of Charlotte Maxeke Johannesburg Academic Hospital (CMJAH), the tertiary referral centre for EGH, and serves as a step down facility for CMJAH. EGH has 230 beds, 40 of which are dedicated to surgical care. ${ }^{13}$

\section{Results}

A total of 95 patients agreed to participate. Six respondents were excluded as they represented extreme outliers (that is, delay to presentation or referral greater than 100 days). These data are summarised in Figure 1. Exclusion of the outliers did not impact on the statistical significance of results.

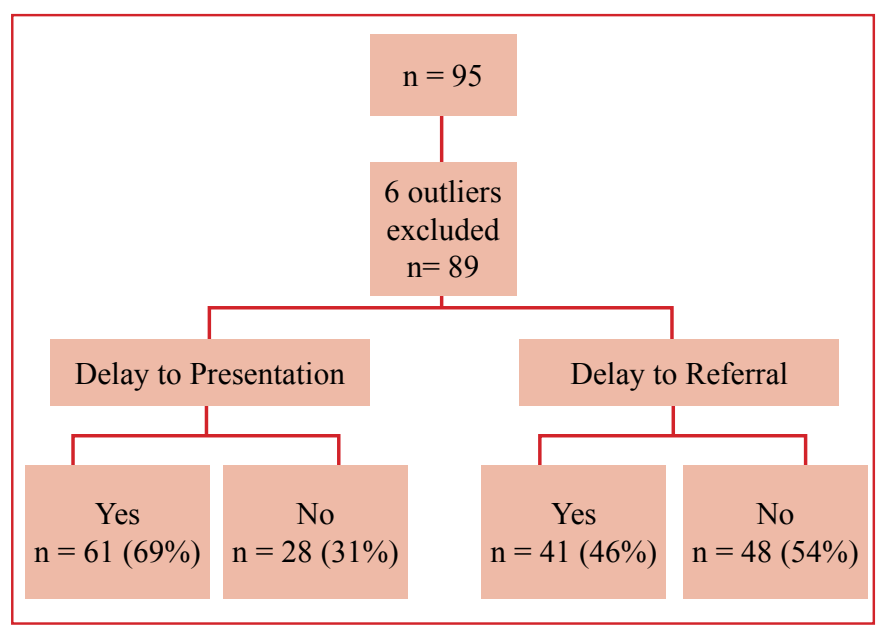

Figure 1: Study CONSORT diagram

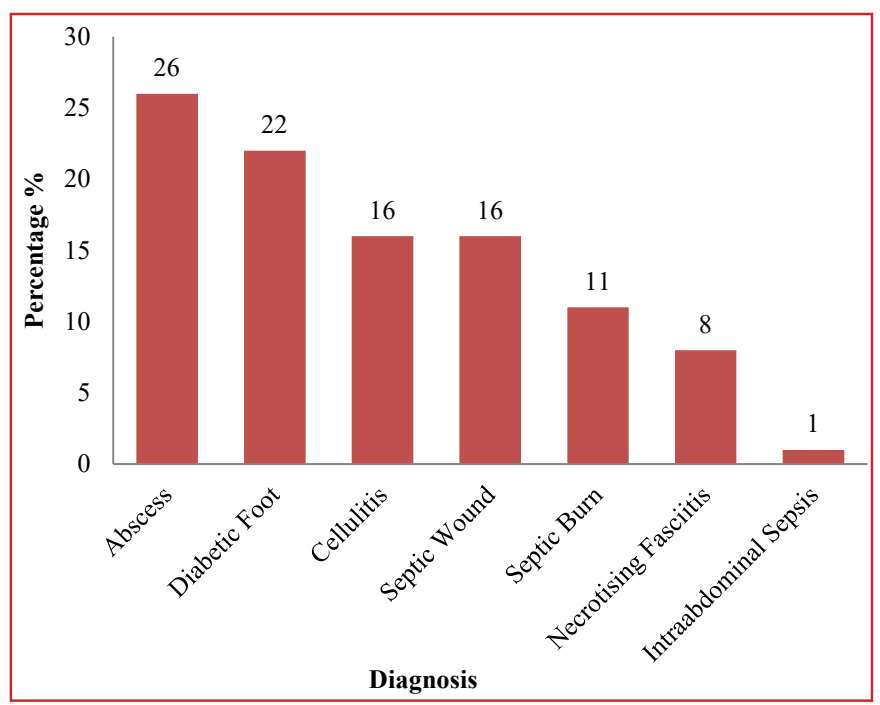

Figure 2: Categories and proportion of admissions

Abscesses (26\%), diabetic feet $(22 \%)$, and cellulitis (16\%) were the most common categories of infection requiring admission. Summary data on diagnoses are presented in Figure 2.

Delayed presentation was noted in $69 \%(61 / 89)$ of patients. Mean delay to presentation was 8.5 days (range 0-90 days, standard deviation 3.8 days). Mean age in the delay to presentation group (51.8 years) was significantly higher $(p=0.019)$ than mean age in the no delay to presentation group (43.5 years). Race, history of diabetes and main source of monthly income were identified as significant categorical variables in delay to presentation. Logistic regression was utilised to determine significant differences between groups within categorical variables identified as significant to delayed presentation and delayed referral. Race was subdivided into Black Africans and non-Black Africans in order to perform logistic regression. No significant difference was found between these groups $(\mathrm{p}=0.088$, OR: $3.8,95 \%$ CI: 0.98 to 25.8 ). Main source of monthly income was the only financial demographic variable identified that significantly 
Table 1: Cohort characteristics

\begin{tabular}{|c|c|c|c|}
\hline Race & & Main Source of Monthly Income & \\
\hline Black & $73(82 \%)$ & Formal Employment & $37(42 \%)$ \\
\hline Coloured & $1(1 \%)$ & Casual Employment & $13(15 \%)$ \\
\hline Asian & $1(1 \%)$ & Contribution of Relatives & $10(11 \%)$ \\
\hline White & $14(16 \%)$ & State Grant & $28(31 \%)$ \\
\hline Housing & & Casual Employment and Contribution of Relatives & $1(1 \%)$ \\
\hline Formal House & $51(57 \%)$ & Monthly Household Income & \\
\hline Informal Dwelling & $7(8 \%)$ & $<\mathrm{R} 1000$ & $20(22 \%)$ \\
\hline Formal Backyard Dwelling & $12(13 \%)$ & R1001-R5000 & $54(61 \%)$ \\
\hline Informal Backyard Dwelling & $4(5 \%)$ & R5001-10000 & $10(11 \%)$ \\
\hline Flat & $12(13 \%)$ & $>\mathrm{R} 10000$ & $5(6 \%)$ \\
\hline Full Time & $31(35 \%)$ & Yes & $1(1 \%)$ \\
\hline Part Time & $7(8 \%)$ & No & $88(99 \%)$ \\
\hline Unemployed & $40(45 \%)$ & Known Diabetic & \\
\hline Piece Job & $10(11 \%)$ & Yes & $34(38 \%)$ \\
\hline Housewife/Husband & $1(1 \%)$ & No & $55(62 \%)$ \\
\hline Household Decision Makers & & Known Retroviral Disease & \\
\hline Self & $53(60 \%)$ & Yes & $14(16 \%)$ \\
\hline Partner & $1(1 \%)$ & No & $66(74 \%)$ \\
\hline Head of household & $8(9 \%)$ & Blank & $9(10 \%)$ \\
\hline Joint Decision & $27(30 \%)$ & First Contact With Health Care Provider & \\
\hline Highest Level of Education & & Local Clinic & $42(47 \%)$ \\
\hline Secondary & $63(71 \%)$ & Edenvale General Hospital & $24(27 \%)$ \\
\hline Tertiary & $7(8 \%)$ & Blank & $1(1 \%)$ \\
\hline Access to Public Transport & & Personal Car & \\
\hline Yes & $60(68 \%)$ & Yes & $17(19 \%)$ \\
\hline No & $19(21 \%)$ & No & $72(81 \%)$ \\
\hline Blank & $10(11 \%)$ & & \\
\hline
\end{tabular}

Table 2: Significant categorical variables in delay to presentation

\begin{tabular}{|c|c|c|c|c|c|}
\hline Category & Category Levels & No Delay n (\%) & Delay n (\%) & $\begin{array}{c}\text { Chi-Squared/ } \\
\text { Fisher's p-Value }\end{array}$ & $\begin{array}{c}\text { Logistic Regression } \\
\text { p-Value }\end{array}$ \\
\hline \multirow{4}{*}{ Race } & Black & $26(29.2 \%)$ & $47(52.8 \%)$ & \multirow{4}{*}{0.012} & \multirow{4}{*}{0.088} \\
\hline & Coloured & 0 & $1(1.1 \%)$ & & \\
\hline & Asian & 0 & $1(1.1 \%)$ & & \\
\hline & White & 0 & $14(15.7 \%)$ & & \\
\hline \multirow{5}{*}{$\begin{array}{l}\text { Main Source } \\
\text { of Monthly } \\
\text { Income }\end{array}$} & Formal Employment & $10(11.2 \%)$ & $27(30.3 \%)$ & \multirow{5}{*}{0.003} & \multirow{5}{*}{0.053} \\
\hline & Casual Employment & $10(11.2 \%)$ & $3(3.4 \%)$ & & \\
\hline & Contribution of Relatives & $3(3.4 \%)$ & $7(7.9 \%)$ & & \\
\hline & State Grant & $5(5.6 \%)$ & $23(25.8 \%)$ & & \\
\hline & $\begin{array}{l}\text { Casual Employment and } \\
\text { Contribution of Relatives }\end{array}$ & 0 & $1(1.1 \%)$ & & \\
\hline \multirow{2}{*}{$\begin{array}{l}\text { Known } \\
\text { Diabetic }\end{array}$} & Yes & $6(6.7 \%)$ & $28(31.5 \%)$ & \multirow{2}{*}{0.034} & \multirow{2}{*}{0.031} \\
\hline & No & $22(24.7 \%)$ & $33(37.1 \%)$ & & \\
\hline
\end{tabular}


impacted on time to presentation $(p=0.03)$. Logistic regression was facilitated by subgrouping source of income as Employed (formal and casual employment) and Unemployed (contribution of relatives and state grant). No significant difference was found between these groups $(\mathrm{p}=0.053$, OR: $2.58,95 \%$ CI: 1.0 to 7.1 ). Whilst personal history of retroviral disease had no impact on time to presentation, personal history of diabetes did. When diabetics were compared with non-diabetics, the odds of diabetics experiencing delay to presentation were 3.1 times greater than those of non-diabetics ( $p=0.031$, OR: $3.1,95 \% \mathrm{CI}: 1.15$ to 9.4 ). These results are summarised in Table 2.

Delayed referral was noted in $46 \%$ (41/89) of respondents. Mean delay to referral was 5.8 days (range 0-60 days, standard deviation 3.0 days). Mean age in the delay to referral group (53.2 years) was significantly higher $(\mathrm{p}=0.025)$ than mean age in the no delay to referral group (45.8 years). Level of first contact with the health care system was the only categorical variable identified as significant in delay to referral. Level of first contact was sub-grouped as presentation to EGH and Other. The odds of patients that initially presented to the local clinic, private physician, or traditional healer (Other) experiencing delayed referral to specialist care were 4.7 times greater than those who first presented to EGH ( $\mathrm{p}=0.005$, OR: $4.7,95 \%$ CI 1.7 to 15.7$)$. These data are summarised in Table 3.

The most common reason for delay given by patients in both the delay to presentation $(84 \%, 51 / 61)$ and delay to referral group $(61 \%, 25 / 41)$ was their belief that their problems would resolve spontaneously. Reasons for delay to care are reported in Table 4.

\section{Discussion}

Abscesses, diabetic foot, and cellulitis were the most common diagnoses requiring surgical management in our series. EGH does not offer an after-hours radiology or surgical service, and this may partially account for the low rate of intraabdominal sepsis. Patients presenting to EGH casualty with suspected intra-abdominal sepsis were likely immediately referred to a higher level of care. The majority of patients in our series were Black African males. El Shallay et al. ${ }^{14}$ and Ahmed et al. ${ }^{15}$ noted a similar male dominance in their study of community acquired abscesses in Sudan and health-seeking behaviour in Bangladesh. Notwithstanding the fact that the majority of women polled in our sample reported being in charge of decisions regarding their health, women still made up a minority of patients. The preponderance for males to preferentially access health care is a well-known phenomenon and applies to various levels of care in developed and developing countries. ${ }^{16}$

More than two thirds of patients experienced a significant delay to presentation. Age, race, main source of monthly income and history of diabetes were significant variables contributing to delay in presentation in the study sample. Research conducted in the United Kingdom and the United States found that White patients were less likely to experience delays to care than Black and female patients. ${ }^{17}$ On subgroup analysis, we did not find gender, race or economic dependency to be significantly related to delay to presentation. This said, the difference between the employed and unemployed groups tended to statistical significance $(\mathrm{p}=0.053)$ in our series. These data imply that the odds of unemployed patients experiencing a delay to presentation are 2.5 times greater than employed patients. Thus, in spite of the availability of free primary care services in the study setting, our findings indicate that some groups may face financial obstacles to access to care in the study sample. In their analysis of barriers to surgical care in LMICs, Ologunde et al. ${ }^{18}$ identify state grants as a structural disincentive to health-seeking behaviour. Ologunde et al. ${ }^{18}$ argue that since eligibility for disability grants is

Table 3: Significant categorical variables in delay to referral

\begin{tabular}{llcccc}
\hline Table 3: Significant categorical variables in delay to referral & & \\
\hline Category & Category Levels & No Delay n (\%) & Delay n (\%) & $\begin{array}{c}\text { Chi-Squared /Fisher's } \\
\text { p-Value }\end{array}$ & $\begin{array}{c}\text { Logistic Regression } \\
\text { p-Value }\end{array}$ \\
\hline & Local Clinic & $21(23.6 \%)$ & $21(23.6 \%)$ & & \\
First Contact & Traditional Healer & 0 & $1(1.1 \%)$ & 0.003 & 0.005 \\
with Health Care & Private Physician & $8(9.0 \%)$ & $14(15.7 \%)$ & & \\
Provider & Edenvale Hospital & $19(21.3 \%)$ & $4(4.5 \%)$ & & \\
& Blank & 0 & $1(1.1 \%)$ & & \\
& & &
\end{tabular}

\begin{tabular}{lll}
\multicolumn{2}{l}{ Table 4: Reasons for delay to presentation and referral } \\
\hline Reason for delay & Delay to presentation, $\mathbf{n}=\mathbf{6 1 ,} \mathbf{( \% )}$ & Delay to referral, $\mathbf{n}=\mathbf{4 1 ,}(\mathbf{\%})$ \\
\hline Thought problem would resolve itself & $51(83.6 \%)$ & $25(60.9 \%)$ \\
Financial constraint & $3(4.9 \%)$ & $3(7.3 \%)$ \\
Transport constraint & $3(4.9 \%)$ & $2(4.8 \%)$ \\
Fear of health care system & $2(3.3 \%)$ & $1(2.4 \%)$ \\
Home responsibilities & $1(1.6 \%)$ & $5(12.2 \%)$ \\
Tried alternate therapy & $0(0 \%)$ & $2(4.9 \%)$ \\
Blank & $1(1.6 \%)$ & $3(7.3 \%)$
\end{tabular}


often related to an individual's disease status, patients with ailments may be reluctant to access available health services in environments characterised by high unemployment where state grants are an important source of personal and household income. Definitive assertions on this aspect of health-seeking behaviour in the local and general populations are not possible from our data, and this issue warrants further inquiry.

One third of the cohort was diabetic. Curiously, history of diabetes was identified as a significant variable contributing to delayed presentation in the study sample. We found the odds of delayed presentation in diabetics to be 3 times greater than non-diabetics. This may be due to numerous factors including inappropriate health education, inappropriate management of diabetes mellitus and its complications at primary care level, and the acceptability of available care for diabetic patients. The notion that deficiencies in primary care increase the risk of poorer surgical outcomes is well supported within existing literature. For instance, in a comparative study between Uganda and the United States, 5-year postoperative survival from breast cancer was lower at a Ugandan tertiary hospital $(56 \%)$ than the United States (82-88\%). Inadequate access to appropriate basic care was found to be one of the reasons for this large discrepancy in outcome. ${ }^{19}$ In their comparative study of surgery in the management of infectious disease in Sweden and South Africa, Jarnheimer et al. ${ }^{4}$ partly attribute the lower frequency of surgery for infectious disease in Sweden to preventative primary care and Sweden's shift to outpatient surgical care. Given the increasing incidence and prevalence of diabetes in South Africa, that diabetic foot was the second most common diagnosis in the cohort, and that diabetic patients are at risk for infection that may require surgical management for multiple reasons, the above findings suggest the need to improve primary care services in the study setting. ${ }^{20,21}$ This may be achieved through up-skilling of care providers and increasing the range of surgical services available at primary care level in the public and private sectors.

Task shifting is an emerging concept in the global public health discourse and has been endorsed by the World Health Organisation (WHO) for the expansion of aspects of surgical care. ${ }^{19,22}$ Task shifting involves the delegation of tasks normally performed by highly qualified to less qualified individuals after an appropriate period of training and supervision. ${ }^{23}$ Although task shifting does highlight concerns over the quality of surgical care provided, it has been shown to be a successful means of addressing surgical need in a variety of different environments. ${ }^{22,24}$ In the study context, task shifting may be utilised as a means to improve the surgical acumen and skills of healthcare workers at the primary care level. In this way, primary care may be reinvigorated in order to appropriately prevent, identify, treat and follow up surgical conditions. ${ }^{19}$ Notwithstanding improvements in care that may be achieved through task shifting, these advances may only be realized and sustained by concurrently addressing infrastructure deficits at primary level..$^{19,22}$

Approximately half of the patients in our study experienced a significant delay from presentation to referral. The odds of delayed referral were greater in patients older than the mean age and those whose first contact occurred at the primary care level (in the public or private sectors). In their study of appendicitis in an urban South African population, Nshuti et al. ${ }^{10}$ found that delay to receipt of appropriate care was partly due to inadequate treatment at primary care level. Kong et al. ${ }^{7}$ made similar findings in their study of appendicitis in rural South Africa. That said, and in contrast to Kong et al., ${ }^{7}$ we did not find lack of transport or patient preference to engage traditional healers to be significant barriers to care. Given the high rate of utilisation of (public and private) primary care services noted in the sample population, the high proportion of patients experiencing delays to referral revealed by our data further highlight deficiencies in the management of surgical sepsis at the primary care level noted in other local studies. ${ }^{7,10}$ Regardless of the quality of surgical care ultimately provided, poor communication between levels of care, inappropriate primary management, late diagnosis and inadequate screening all adversely affect the outcomes of surgical care. ${ }^{19}$

In their study of health seeking behaviour in Bangladesh, Ahmed et al. ${ }^{15}$ found that symptom recognition does not automatically result in presentation to a health care facility. The most common reason given by patients in the sample population for delay to presentation and referral was their perception that their problems would resolve spontaneously. Given that the overwhelming majority of patients in our sample made their own decisions regarding personal health, this may suggest that acceptability of care is a significant barrier to seeking care in our environment. Akande et al., ${ }^{25}$ in their study of health-seeking behaviour in Anyigba, Nigeria, found that more than half of the patients in their series experienced a delay to care due to the assumption that their problems would resolve spontaneously. Given the high proportion of patients with delay to presentation and referral in our series, there is a need to increase health education in communities. In conjunction with broader initiatives such as community health education, relatively simple interventions such as the provision of regular transport from primary care centers to higher level facilities may directly influence time to referral. This is confirmed by a study performed in India, which demonstrated that the provision of transport services significantly increased uptake of services for cataract surgery. ${ }^{26}$

Given that many of the barriers to care found in our study are interlinked, we believe that delays to care may be addressed by a multifaceted intervention strategy addressing the affordability, accessibility, availability and acceptability of health care services. More specifically, these interventions may include: (i) health campaigns that specifically target women in order to improve their utilization of health care services, (ii) education programmes on diabetes mellitus, its management and complications for both health care workers at primary level and patients with diabetes mellitus, (iii) improvements in the quality of primary care services that address infrastructural and resource deficiencies and skills deficits, and (iv) interventions to improve the efficiency of referral from primary to higher levels of care. Future inquiry 
into barriers to care for patients with infections requiring surgical management may focus on numerous other areas. These include but are not limited to: (i) the perceptions of health care workers on barriers to care at primary and higher levels of care, (ii) assessment of the knowledge of and resources available to primary care workers to manage infectious surgical pathology and chronic disease with surgical complications, and (iii) a review of existing health promotion programmes within the community serviced by EGH.

\section{Limitations}

This was a prospective descriptive study performed by means of convenience sampling at a single centre with variable data collection over a two year period. The study was limited by its sample size and distribution of respondents over time. Further, and due to the nature of the study design, results may have been influenced by selection, responder and recall bias. No record was kept of the number of patients that declined study involvement, and this may have contributed to selection bias. Given the small sample size, analysis of categories within the population may have been prone to type II errors. Collection of morbidity and mortality data would have facilitated investigation between delay to care and patient outcome. Unfortunately outcome data was not collected. Gaps in data collection over the study period due to time and resource constraints limit the validity of the findings. Future investigation that accounts for the limitations of this study will improve the validity of findings.

\section{Conclusions}

The majority of respondents were unemployed, Black African males. Skin and soft tissue infections were the most common diseases requiring surgical management. Delay to presentation and referral was noted in nearly half of all patients. Patients' socio-economic status, past medical history, age, level of first contact with the health care system, and perceptions of their own health were found to contribute to delays in seeking and receiving care in the study sample. Given that many of the barriers to care found in our study are interlinked, we believe that delays to care may be addressed by a local multifaceted intervention strategy. Given its limitations this study is hypothesis generating, and requires adequately designed future studies to ensure external validity of findings.

\section{Acknowledgements}

The authors would like to thank Dr J Witt, Dr S Birtles, and C Naidoo for their contribution.

\section{REFERENCES}

1. Lever A, Mackenzie I. Sepsis: definition, epidemiology and diagnosis. BMJ. 2007;335(7625):879-83. Available at: http:// dx.doi.org/10.1136/bmj.39346.495880.AE

2. Kumar A, Roberts D, Wood KE, et al. Duration of hypotension before initiation of effective antimicrobial therapy is the critical determinant of survival in human septic shock. Crit Care Med.
2006;34(6):1589-96. Available at: http://dx.doi.org/10.1097/01. CCM.0000217961.75225.E9

3. Buck DL, Vester-Andersen M, Moller MH. Surgical delay is a critical determinant of survival in perforated peptic ulcer. Br J Surg. 2013;100:1045-9. Available at: http://dx.doi.org/10.1002/ bjs. 9175

4. Jarnheimer A, Kantor G, Bickler S, et al. Frequency of surgery and hospital admissions for communicable diseases in a high- and middle-income setting. Br J Surg 2015;102:1142-9. Available at: http://dx.doi.org/10.1002/bjs.9845

5. Ozgediz D, Riviello R. The "Other" Neglected Diseases in Global Public Health: Surgical Conditions in Sub-Saharan Africa. PLoS Med. 2008;5(6):e121. Available at: http://dx.doi. org/10.1371/journal.pmed.0050121

6. Poenaru D, Pemberton J, Cameron BH. The burden of waiting: DALYs accrued from delayed access to pediatric surgery in Kenya and Canada. J Pediatr Surg. 2015;50(5): 765-70. Available at: http://dx.doi.org/10.1016/j.jpedsurg.2016.02.033

7. Kong VY, Bulajic B, Allorto NL, et al. Acute appendicitis in a developing country. World J Surg. 2009;36(9):2068-73. Available at: http://dx.doi.org/10.1007/s00268-012-1626-9

8. Mansouri A, Chan V, Njaramba V, et al. Sources of delayed provision of neurosurgical care in a rural Kenyan setting. Surg Neurol Int. 2015;25(6):e32. Available at: http://dx.doi. org/10.4103/2152-7806.152141

9. Meara JG, Leather AJ, Hagander L, et al. Global Surgery 2030: evidence and solutions for achieving health, welfare, and economic development. Lancet. 2015;386(9993):569624. Available at: http://dx.doi/org/10.1016/S01406736(15)60160-X

10. Nshuti R, Kruger D, Luvhengo T. Clinical presentation of acute appendicitis in adults at the Chris Hani Baragwanath Academic Hospital. Int J Emerg Med. 2014;7:12. Available at: http:// dx.doi.org/10.1186/1865-1380-7-12

11. Mock CN, Donkor P, Gawande A, et al. Essential surgery: key messages from Disease Control Priorities 3rd Ed. Lancet. 2015;385(9983):2209-19. Available at: http://dx.doi. org/10.1016/S0140-6736(15)60091-5

12. Mathee A, Barnes B, Naicker N, et al. The Health, Environment and Development Study Preliminary Results of Phase One. A report prepared for the World Health Organisation Collaborating Centre for Urban Health. Johannesburg: South African Medical Research Council. 2006. Available at: www.mrc.ac.za/ environmenthealth/whoheadrptpart1.pdf

13. Kernes N. Personal Communication. CEO Edenvale Hospital; 2014.

14. El Shallaly GH, Hassan AN, Siddig NO, et al. Study of patients with community-acquired abscesses. Surg Infect (Larchmt). 2012;13(4):250-6. Available at: http://dx.doi.org/10.1089/ sur.2011.069

15. Ahmed SM, Adams AM, Chowdhury M, et al. Gender, socioeconomic development and health-seeking behaviour in Bangladesh. Soc Sci Med. 2000;51(3):361-71. Available at: http://dx.doi.org/10.1016/S0277-9536(99)00461-X

16. Isaacs-Long Y, Myer L, Zar H. Trends in admissions, morbidity and outcomes at Red Cross War Memorial Children's Hospital, Cape Town, 2004-2013. S Afr Med J. 2017;107(3):21926. Available at: http://dx.doi.org/10.7196/SAMJ.2017. v107i3.11364

17. Adamson J, Ben-Shlomo Y, Chaturvedi N, et al. Ethnicity, socio-economic position and gender - do they affect reported health-care seeking behaviour? Soc Sci Med. 2003;57(5): 895-4. Available at: http://dx.doi.org/10.1016/S0277-9536(02)00458-6 
18. Ologunde R, Maruthappu M, Shanmugarajah K, et al. Surgical care in low and middle-income countries: Burden and barriers. Int J Surg. 2014;12:858-63. Available at: http://dx.doi org/10.1016/j.ijsu.2014.07.009

19. Vasan A, Hudelson CE, Greenberg SL, et al. An integrated approach to surgery and primary care strengthening in low- and middle-income countries: Building a platform to deliver across the spectrum of disease. Surgery. 2015;157(6):965-70. Available at: http://dx.doi.org/10.1016/j.surg.2015.03.003

20. Casqueiro J, Casqueiro C, Alves C. Infections in patients with diabetes mellitus: A review of pathogenesis. Indian J Endocrinol Metab. 2012;16(Supp11):S27-S36. Available at: http://dx.doi org/10.4103/2230-8210.94253

21. SEMDSA Type 2 Diabetes Guidelines Expert Committee. SEMDSA 2017 Guidelines for the Management of Type 2 diabetes mellitus. JEMDSA. 2017;22(1)(Supp11):S1-S196. Available at: www.jemdsa.co.za

22. Ologunde R, Maruthappu M, Shanmugarajah K, et al. Surgical care in low and middle-income countries: Burden and barriers. Int J Surg. 2014;12:858-63. Available at: http://dx.doi. org/10.1016/j.ijsu.2014.07.009

23. Okyere E, Mwanri L, Ward P. Is task-shifting a solution of health workers' shortage in Northern Ghana? PLoS One. 2017;12(3):e0174631. Available at: http://dx.dor.org/10.1371/ journal.pone. 0174631

24. Atiyeh BS, Gunn SW, Hayek SN. Provision of essential surgery in remote and rural areas of developed as well as low and middle income countries. Int J Surg. 2010;8:581-5. Available at: http://dx.doi.org/10.1016/j.ijsu.2010.07.291

25. Akande TM, Owoyemi JO. Healthcare-Seeking Behaviour in Anyigba, North-Central, Nigeria. Res J Med Sci. 2009;3(2):4751.

26. Grimes CE, Bowman KG, Dodgion CM, et al. Systematic review of barriers to surgical care in low-income and middleincome countries. World J Surg. 2011;35:941-50. Available at: http://dx.doi.org/10.1007/s00268-011-1010-1 